\title{
Deriving the Vehicle Speeds from Mobile Telecommunications Network
}

\author{
Ren-Huang Liou, Yi-Bing Lin, Fellow, IEEE, Yu-Long Chang \\ Department of Computer Science \\ National Chiao Tung University \\ \{rhliou, liny, ylchang\}@cs.nctu.edu.tw \\ Hui-Nien Hung, Nan-Fu Peng \\ Institute of Statistics \\ National Chiao Tung University \\ \{hhung, nanfu\}@stat.nctu.edu.tw \\ Ming-Feng Chang \\ Department of Computer Science \\ National Chiao Tung University \\ mfchang@cs.nctu.edu.tw
}

\begin{abstract}
Vehicle speeds of roads are often measured by the Intelligent Transportation Systems (ITS) through some sensors or software solutions. Our previous work proposed the LinChang-Huangfu (LCH) scheme to compute the cell residence times by the standard counter values in the mobile telecommunications switches. In this paper, we use mathematical and statistical developments to investigate the accuracy of the LCH scheme by deriving the bias of the cell residence times computed in this scheme. Then we extend the LCH scheme with
\end{abstract}


some filtering and compensation techniques for vehicle speed estimation, and validate our approach with vehicle detector measurements at National Highway 3, Longtan Township, Taoyuan County, Taiwan. Our study indicates that the LCH scheme is an effective approach for the vehicle speed estimation.

\section{Index Terms: Lin-Chang-Huangfu (LCH) Scheme, Mobile Switching Center (MSC), telecommunication, vehicle speed}

\section{Introduction}

Most Intelligent Transportation Systems (ITS) measure the vehicle speeds of the roads to assist vehicle drivers to estimate the travel times and to avoid the traffic jam. To provide this service, an ITS server is responsible for collecting and computing the vehicle speeds. This traffic information can be accessed by the users through networks such as the Internet.

The ITS server can obtain the traffic information from the mobile telecommunications network. The intuition behind this approach is described as follows. When you run faster, you pass telephone poles on the side of the road more frequently. Similarly, when a car travels down a road, it will pass cell phone towers (base stations or BSs) more often. The length of time a cell phone is connected to a particular BS will vary inversely with speed. In fact, the speed of the vehicle can be estimated just by dividing the length of the road covered by a particular BS by the difference between the time when it leaves the cell (radio coverage of the BS) and when it entered that cell.

Information about when "handing over" from one BS to another is available in the mobile telecommunications network, so the speed can be estimated if the intersections of cells relative to the road travelled on are also known. Of course, the vehicle itself already has more accurate means of estimating its velocity, but the speedometer readings are not typically accessible externally. Also, Global Positioning System (GPS) provides a much more 
accurate method of estimating speed, but running GPS continuously takes a lot of power, and some cell phones do not even have a GPS receiver. The alternate method based on frequency of handovers could provide speed estimation for map-based information on traffic to other travelers. Such a system would require installation of a central server (i.e., the ITS server) to gather all the information and to make the resulting summary traffic information available over the Internet. These statistics include (i) the number of handovers into each cell, (ii) the number of handovers out of each cell, and (iii) the total voice traffic. Here voice traffic is measured as the sum of the call holding times for all calls.

Our previous work [1-3] proposed the Lin-Chang-Huangfu (LCH) scheme to estimate the cell residence time (the time periods that the UE stays in the cells) by the ratio of the voice traffic and the number of handovers into the cell. The cell residence time in turn can be used to estimate the speed as above. This paper is a major extension of our previous conference paper [4]. We compute the cell residence times of LCH to estimate the vehicle speeds, and validate the LCH scheme with the vehicle detector measurements at National Highway 3, Longtan Township, Taoyuan County, Taiwan. A major contribution of this paper is the investigation of the bias for the cell residence times computed by the LCH scheme. This kind of bias derivation has not been found in the literature, which shows that the LCH scheme is an appropriate approach for vehicle speed estimation.

This paper is organized as follows. Section 2 describes the related work. Section 3 describes how the LCH scheme computes the cell residence times. Section 4 derives the bias of the cell residence times computed by the LCH scheme. Section 5 proposes several techniques for improving the accuracy of the vehicle speed estimation. Section 6 investigates the performance of the LCH scheme by numerical examples, and the conclusions are given in Section 7. 


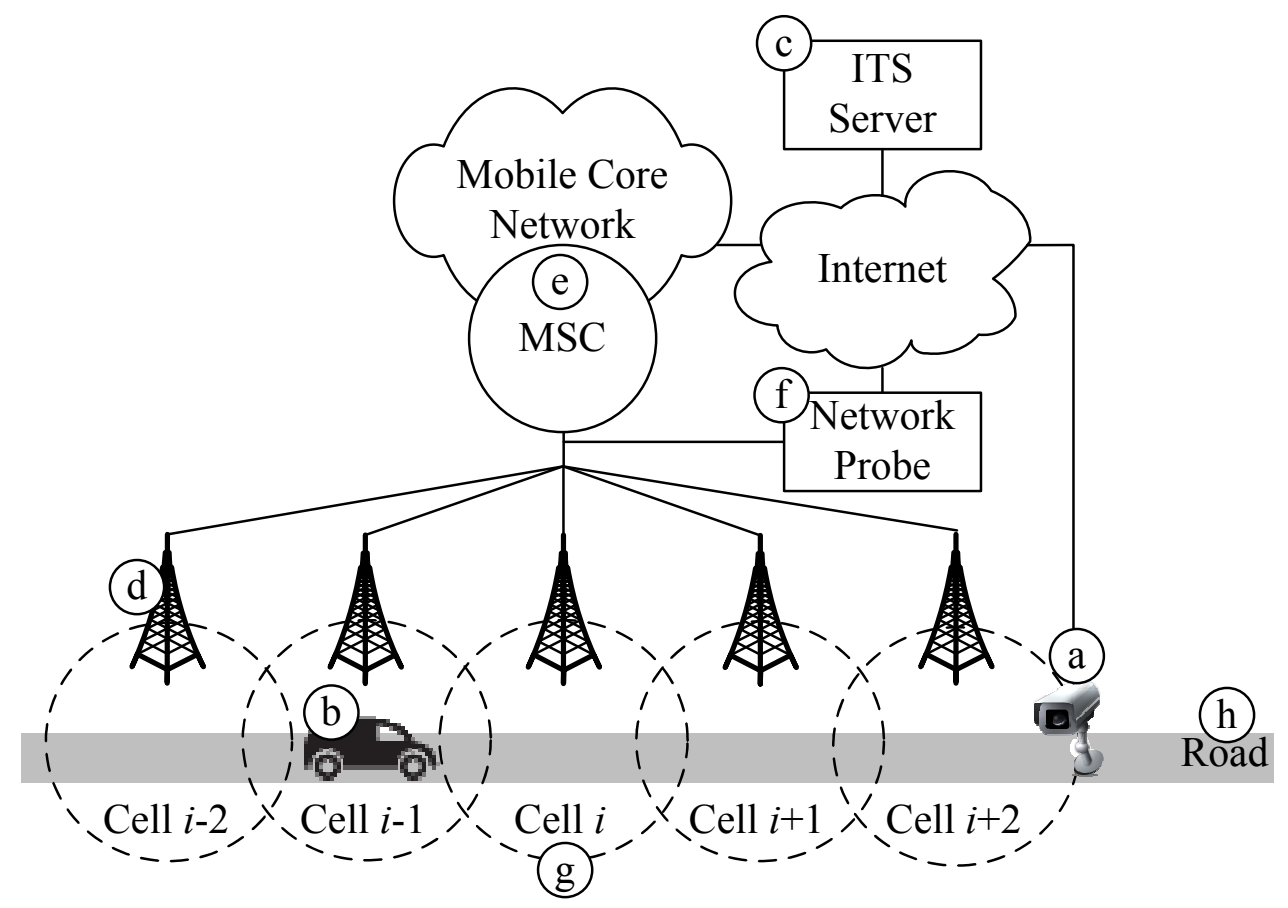

Figure 1: A Simplified Mobile Telecommunications Network Architecture

\section{Related Work}

The vehicle speed measurement approaches can be classified into three categories:

- Vehicle Detector (VD) [5]: the VDs (Figure 1 (a)) are installed in the roads to measure the speeds of the vehicles (Figure 1 (b)), and the speeds are reported to the ITS server (Figure 1 (c)) through a wireline or a wireless network.

- GPS-based Vehicle Probe (GVP) [6,7]: the User Equipments (UEs; i.e., the mobile devices) in the vehicles are equipped with GPS receivers, which send the GPS coordinates and time information through the mobile telecommunications network (i.e., the Base Stations or BSs in Figure 1 (d) and Mobile Switching Center or MSC in Figure 1 (e) to the ITS server. The ITS server computes the speeds according to the received GPS coordinates. 
- Cellular Floating Vehicle Data (CFVD) [8-10,16,17]: the network probes (Figure 1 (f) are installed to monitor the signals between the BSs and the MSC, and send them to the ITS server. The network probe also replaces the user identities and the phone numbers in the signals by their hash values from the one-way hash function to protect the user privacy [18]. Based on the call activities of the UEs, the ITS server tracks the locations of the UEs at the cell level, and estimates the cell residence times of the UEs to derive their moving speeds.

The VD approach is typically deployed by the transportation department of the government. On the other hand, the GVP and CFVD approaches are typically developed by the telecommunication operators. The VD approach suffers from high construction and maintenance costs for detectors $[8,10]$. The GVP approach requires that the UEs are equipped with the GPS receivers, and consumes extra radio resources to transmit the GPS data. Furthermore, our experience (with Chunghwa Telecom) indicates that not many vehicles with GPS receivers travel in some suburban/country roads, and the traffic information for these roads is difficult to obtain from the GPS data. Therefore, the GVP approach is typically used in urban areas. For suburban/country areas, the vehicle speeds are obtained by the CFVD approach, which requires extra signaling links and network probes in the mobile telecommunications network to monitor the signaling messages delivered between the UEs and the mobile core network.

Our previous work [1-3] proposed the Lin-Chang-Huangfu (LCH) scheme, an enhancement of the CFVD approach that does not require extra hardware to collect the data as the CFVD does. The LCH scheme derives the cell residence times from the standard counter values that are automatically and periodically collected by the MSCs. The details of the LCH scheme will be described in Section 3. 


\section{Lin-Chang-Huangfu (LCH) Scheme}

The LCH scheme was described in [1], and the details are re-iterated here for the reader's benefit. In Figure 1, the MSC (Figure 1 (e) is responsible for the call processing and mobility management [11]. The MSC is connected to a group of BSs. The radio coverage of a BS is a cell (see the dashed circles; Figure 1 (g)). During a phone conversation, the UE in a cell connects to the MSC through the BS. If the UE in conversation moves from one cell to another, then the call path is switched from the old cell to the new cell. This process is referred to as handover.

In standard commercial mobile telecommunications operation, the MSC records the call activities of the UEs (e.g., when the UE makes/receives a call or when the UE in conversation hands over from one cell to another). The MSC collects the statistics of the activities in each cell for every $\Delta t$ interval typically ranging from 15 minutes to several hours. Two of the statistics are the number of handovers in and out of the cells and the voice traffic (in Erlang)

of the cells. Consider a time point $\tau$. We define $\Delta \tau$ as the timeslot $\left(\left\lfloor\frac{\tau}{\Delta t}\right\rfloor \Delta t,\left\lfloor\frac{\tau}{\Delta t}+1\right\rfloor \Delta t\right)$. For the description purpose, we define the road segment for speed estimation as target road segment (Figure 1 (h)). The average cell residence time of cell $i$ (Figure 1 (g)) is derived as follows. Let $\rho(\tau)$ be the carried traffic of cell $i$ in $\Delta \tau$. In other words, $\rho(\tau)$ is the number of calls arriving at cell $i$ in $\Delta \tau$ times the expected carried call holding times (measured in minutes). The carried call holding time is different from the offered call holding time. The offered call holding time is the duration (in minutes) of a call if there were unlimited radio channels in all $\mathrm{BSs}$, and the new calls and the handovers are always successful. In reality, the capacity of a BS is limited, and therefore a call attempt may be blocked at the beginning or may be forced to terminate in a handover. For a call that is connected, the call minutes are actually measured at the MSC in the $\rho(\tau)$ statistics, and are defined as the carried call holding time $t_{c}$. In the duration $t_{c}$ of a carried call measured in the MSC, the call is not blocked at the beginning and is not forced to terminate at handovers occurring in 
$t_{c}$ (although it may be forced to terminate at the end of $t_{c}$ ). Let $\gamma(\tau)$ denote the number of handovers into cell $i$ in $\Delta \tau$. Let $t_{m}$ be the cell residence time. In [1], we estimated the cell residence time $t_{m}^{*}(\tau)$ of the UE arriving at cell $i$ in timeslot $\Delta \tau$ as

$$
t_{m}^{*}(\tau)=\frac{\rho(\tau)}{\gamma(\tau)}
$$

Based on (1), the average vehicle speed of the one-way target road segment is derived as follows. Suppose that the vehicle with the UE (Figure 1 (b)) in cell $i-1$ moves to cell $i+1$ through cell $i$. Let $x$ be the length of the target road segment covered by cell $i$. From (1), the average vehicle speed $v(\tau)$ of the target road segment in $\Delta \tau$ can be computed as

$$
v(\tau)=\frac{x}{t_{m}^{*}(\tau)}=\frac{x \gamma(\tau)}{\rho(\tau)}
$$

Now consider a two-way road. To compute the average speed of each direction in the twoway road, we first determine the moving directions of the UEs by their handover sequences of cells. Then based on the moving directions, we compute $\gamma(\tau)$ and $\rho(\tau)$ of each direction. The average speed of each direction is computed by $\gamma(\tau)$ and $\rho(\tau)$ of each direction by using Equation (2).

In the next section, we derive the bias of the cell residence times computed by the LCH scheme. The bias will indicate that the LCH scheme is a good meter for estimating the vehicle speeds.

\section{The Bias of the Cell Residence Time Estimation}

This section evaluates the accuracy of the LCH scheme by estimating the bias of this scheme. We will show that the LCH scheme has smaller bias for the vehicle speed estima- 
tion than the general cell residence time estimation, and therefore the LCH scheme is an appropriate approach to estimate the vehicle speeds.

Let $t_{m}$ be the real cell residence time, and $t_{m}^{*}(\tau)$ be the estimator of $t_{m}$. Then the bias of the cell residence time estimator is defined as

$$
b_{t_{m}}\left(t_{m}^{*}(\tau)\right)=E\left[t_{m}^{*}(\tau)\right]-E\left[t_{m}\right]
$$

Note that in statistics, the bias and the error are not the same. The bias of an estimator is the difference between the estimator's value and the real value [12]. On the other hand, the error is the discrepancy between an exact value and its approximation. In the rest of this section, we first derive $\gamma(\tau)$ and $\rho(\tau)$ constrained by $\Delta \tau$. Then we express the cell residence time estimator $t_{m}^{*}(\tau)$ by the derived $\gamma(\tau)$ and $\rho(\tau)$. Finally, $(3)$ is used to compute the bias.

Figure 2 illustrates the timing diagram of the activities of 6 UEs in an observation time period $\left[t_{0}, t_{19}\right]$. For example, consider the behavior of UE 3 . UE 3 moves to cell $i$ at $t_{8}$ (marked by $\triangle$ ), and a call is connected to UE 3 at $t_{11}$ (marked by $\circ$ ). UE 3 leaves cell $i$ at $t_{13}$ (marked by $\boldsymbol{\Delta}$ ) and the call for UE 3 completes at $t_{15}$ (marked by $\bullet$ ). The cell residence time for UE 3 is $t_{m}=t_{13}-t_{8}$. The carried call holding time for UE 3 is $t_{c}=t_{15}-t_{11}$.

Consider timeslot $\Delta \tau=t_{19}-t_{5}$ (see the gray area in Figure 2). A call observed in cell $i$ in $\Delta \tau$ can be one of the following three types:

- A new call that is originated in $\Delta \tau$; e.g., the call arrivals at time $t_{10}$ for UE 6 , and $t_{11}$ for UE 3 in Figure 2.

- An existing call that is already connected in cell $i$ at the beginning of $\Delta \tau$; e.g., the call for UE 1 originated at $t_{4}$ before timeslot $\Delta \tau$ when UE 1 was in cell $i$; in other words, UE 1 arrived at cell $i$ before $t_{5}$, and the call started in the cell at $t_{4}<t_{5}$ is still in progress. 


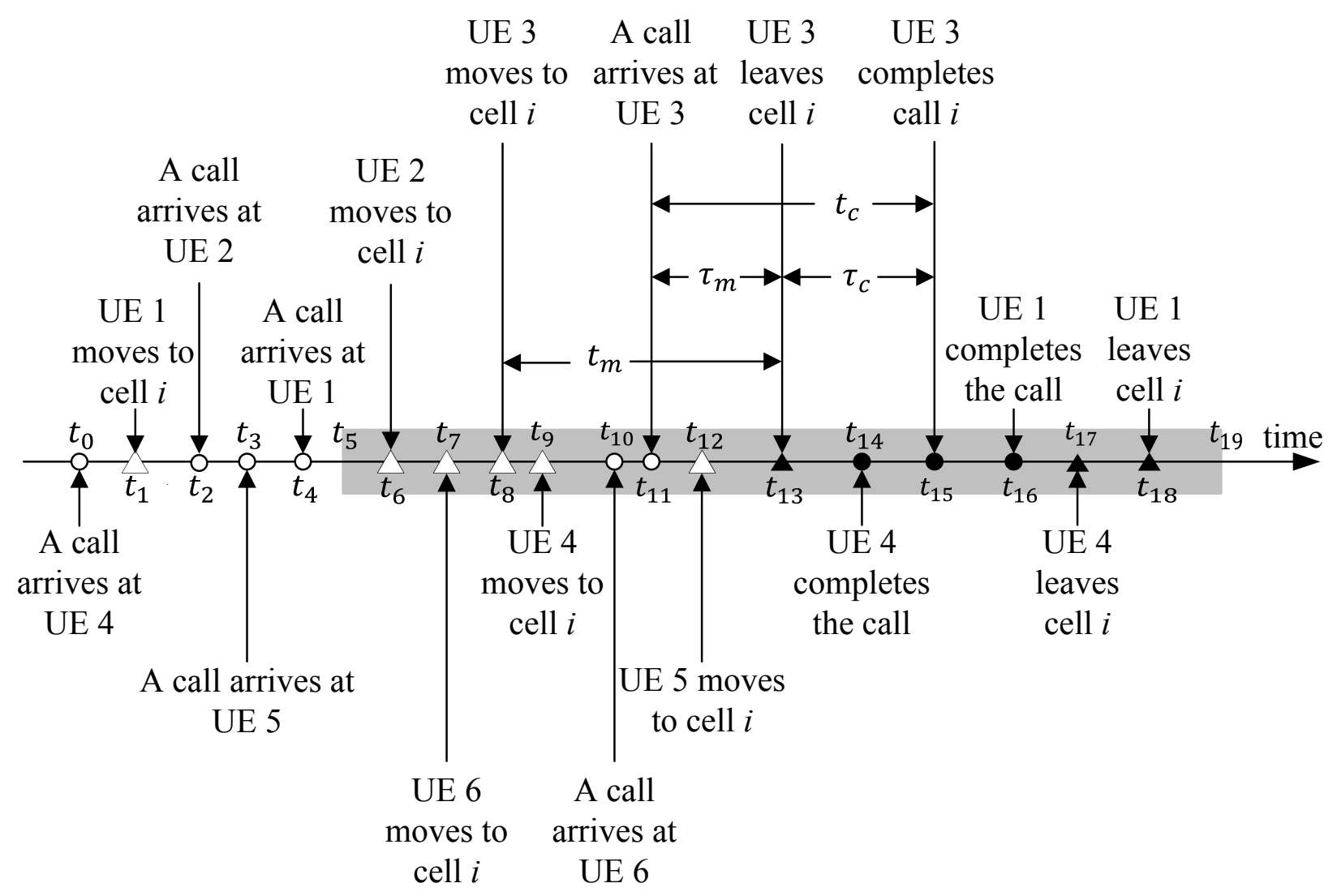

Figure 2: The Timing Diagram for UE Movements and Call Arrivals ( $\triangle$ : an UE moves to cell $i, \boldsymbol{\Delta}$ : an UE leaves cell $i$, ०: a call arrives, $\bullet$ : a call completes, $\square: \Delta \tau)$

- A handover call that is switched from another cell to this cell; e.g., the handovers at time $t_{6}$ for UE $2, t_{9}$ for UE 4 and $t_{12}$ for UE 5 .

Let $\alpha(\tau), \beta(\tau)$ and $\gamma(\tau)$ be the numbers of new calls, existing calls, and handover calls in $\Delta \tau$ in cell $i$, respectively. In Figure $2, \alpha(\tau)=2$ (i.e., the calls originated at $t_{10}$ and $t_{11}$ ), $\beta(\tau)=1$ (i.e., the call originated at $t_{4}$ ), and $\gamma(\tau)=3$ (i.e., the calls handed over at $t_{6}, t_{9}$, and $\left.t_{12}\right)$. Let $\lambda$ be the call arrival rate. It is clear that

$$
E[\alpha(\tau)]=\lambda \Delta \tau
$$

Let $t_{m}$ be a random variable with the mean $\frac{1}{\eta}$, and $t_{c}$ be a random variable with the mean $\frac{1}{\mu}$. In Appendix A, we prove 5 facts which lead to the following important theorem. 
Theorem 1. Assume that $t_{c}$ and $t_{m}$ have arbitrary distributions with the means $\frac{1}{\mu}$ and $\frac{1}{\eta}$, respectively. If timeslot $\Delta \tau=\frac{1}{\delta}$ is fixed, and we observe the call activities for a long period $t$, then

$$
b_{t_{m}}\left(t_{m}^{*}(\tau)\right)=\frac{\mu \delta}{\lambda \eta^{2}}
$$

Equation (5) indicates that the LCH scheme has better accuracy when the call holding time is long (i.e., $\mu$ is small), $\Delta \tau$ is long (i.e., $\delta$ is small), the call arrival rate $\lambda$ is large, or the cell residence time is short (i.e., $\eta$ is large). Because the vehicles typically have much shorter cell residence times (i.e., higher speeds) than the pedestrians, equation (5) indicates that the LCH scheme has higher accuracy in estimating the vehicle speeds than the general cell residence time estimation that also includes pedestrians.

\section{Techniques for Improving the Accuracy of the Vehi- cle Speed Estimation}

This section describes several techniques to further improve the accuracy of the vehicle speed estimation expressed in (2). We first introduce two filtering techniques to exclude the UEs not in the target road segment. Then we describe two compensation techniques to increase the number of observed calls based on the leaky-bucket integration strategy [15]. Note that these techniques may not cover all realistic road configurations. The purpose of this section is to demonstrate that the accuracy of speed estimation can be improved by the concepts of "filtering" and "compensation". Based on the filtering/compensation concepts, we can further develop other potential techniques to improve the accuracy, and hope that the readers of this paper can take these concepts to develop appropriate techniques in their scenarios. 


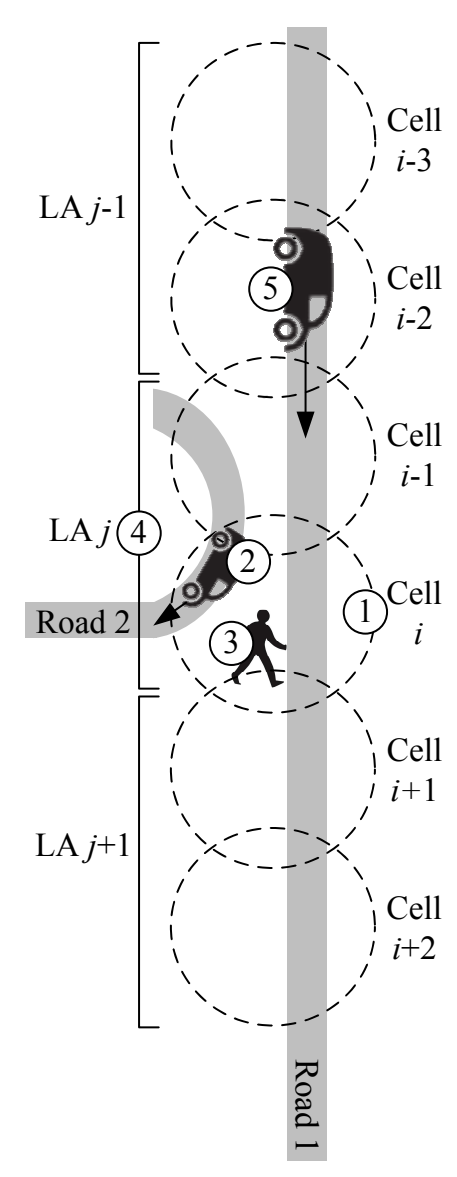

(a) A Cell may Cover Several Roads and Pedestrians

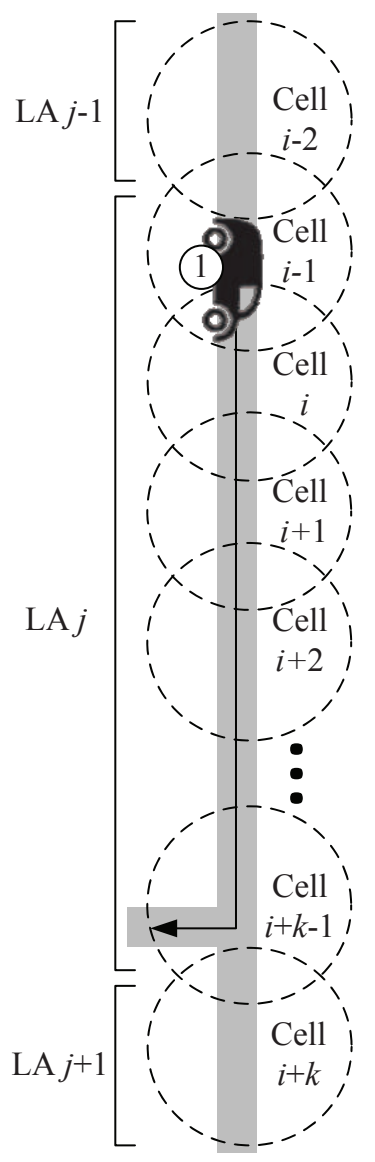

(b) A Vehicle may Move to Another Road without Leaving the LA of a Cell ( $k=16$ in our experiment environment)

Figure 3: Two Scenarios that Affect the Accuracy of the Vehicle Speed Estimation

If cell $i$ (1) in Figure 3 (a)) also covers the area other than the target road segment (2) and (3) in Figure $3(\mathrm{a})), \gamma(\tau)$ and $\rho(\tau)$ also include the call activities of the UEs not in the target road segment. These activities may reduce the accuracy of (2). To resolve this issue, we first introduce the standard location update procedure in mobile telecommunications network, and then show how to identify the UEs in the target road segment from the UEs outside the target road segment but in cell $i$. In mobile telecommunications network, the cells are grouped into Location Areas (LAs; e.g., LA $j$ contains cells $i-1$ and $i$; see (4) in Figure 3 (a)). When a UE (5) in Figure 3 (a)) moves from one LA to another, the UE executes the location update procedure to inform the MSC of its new LA $[11,14]$. The location update 
messages are delivered from the BS to a mobility database (specifically, Visitor Location Register or VLR) through the MSC. Based on the location update, we propose the following technique to identify the UEs in the target road segment:

Filtering Technique 1. For every UE which has call activities in cell $i$, let cell $A$ be the cell where the UE performs the location update when entering the LA of cell $i$, and let cell $B$ be the cell where the UE performs another location update when leaving the LA of cell $i$. If both cells $A$ and $B$ cover the road, then the UE is identified as in the target road segment.

For example, when the UE moves from LA $j-1$ to LA $j+1$ through LA $j$ (see (5) in Figure 3 (a)), one location update is performed in cell $i-1$ of LA $j$ (i.e., cell $A$ ), and another location update is performed in cell $i+1$ of LA $j+1$ (i.e., cell $B$ ). Because both cells $i-1$ and $i+1$ cover the road, the UE is identified as a vehicle moving in the target road segment.

Depending on their destinations, some vehicles may be stopped within the LA of cell $i$, or move from the target road to another road (see (1) in Figure 3 (b)). Those UEs may not be identified by filtering technique 1 , but can be detected by the following technique:

Filtering Technique 2. For every UE which has the call activities in cell $i$, if the UE's handover sequence of cells contains at least three cells which cover the road, the UE is identified in the target road segment.

In Figure 3 (b), if the UE's handover sequence of cells contains cells $\{i-2, i-1, i\}$, cells $\{i-1, i, i+1\}$, or cells $\{i, i+1, i+2\}$, the UE is identified in the target road segment.

For the UEs identified by filtering techniques 1 or 2 , we use their handover information and call holding times in cell $i$ to compute $\gamma(\tau)$ and $\rho(\tau)$ of the target road segment. Then the average speed of the target road segment is computed by using (2). 
Although these two filtering techniques effectively exclude the UEs not in the target road segment, they also reduce the number of the observed calls. It is clear that if few calls are observed in $\Delta \tau$, the samples cannot reflect the actual vehicle speeds of the target road segment. To resolve this issue, we consider the far-history and near-history compensation techniques based on the leaky-bucket integration strategy. The far-history compensation technique uses the "same $\Delta \tau$ " on the same days of the past weeks to compensate for the number of the observed calls. On the same days of the past weeks, the traffic patterns are typically similar; e.g., the traffic patterns of this Monday are similar to those of last Monday. Similarly, for national holidays (e.g., the new year day), we will use far-history compensation of the last national holiday (e.g., the last new year day). For timeslot $\Delta \tau$, let $\Delta \tau_{k}$ be the same timeslot on the same day of the most recent $k$ th week, and let $\Delta \tau_{0}=\Delta \tau$. For example, if $\Delta \tau$ is the timeslot on this Monday, then $\Delta \tau_{1}$ is the same timeslot on last Monday. The far-history compensation technique guarantees that $v(\tau)$ is computed by at least $K_{h}$ samples of handovers. The details are given below.

Far-History Compensation Technique. The average speed of the target road segment with the threshold $K_{h}$ is computed as

$$
v(\tau)=\frac{x\left[\sum_{k=0}^{K} \gamma\left(\tau_{k}\right)\right]}{\sum_{k=0}^{K} \rho\left(\tau_{k}\right)} \text { where } K=\min \left\{N: \sum_{n=0}^{N} \gamma\left(\tau_{n}\right) \geq K_{h}\right\}
$$

Note that in (6), if $\gamma(\tau)$ is no less than $K_{h},(6)$ is the same as (2) (i.e., no historical datum is used).

Because the MSC collects $\rho(\tau)$ and $\gamma(\tau)$ for every $\Delta t$ interval, some calls may cross the timeslot (i.e., the existing calls). These "crossing-timeslot" calls may cause non-smooth effect on the consecutive timeslots. The leaky-bucket strategy can smooth the results if the traffic patterns of the consecutive timeslots do not significantly change. However, if the speeds of the consecutive timeslots dramatically change (e.g., the difference between these 


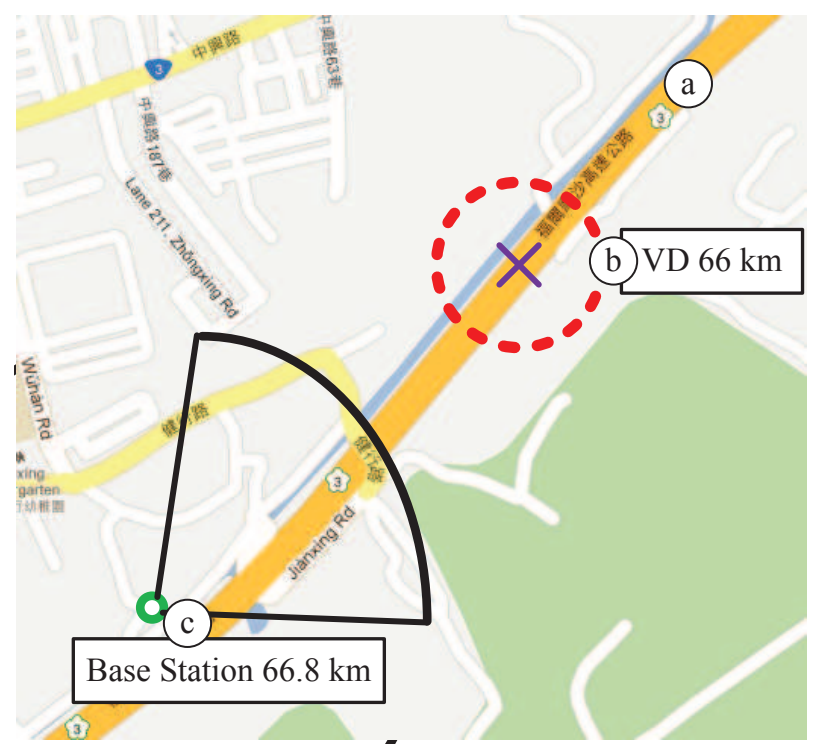

Figure 4: The Experimental Environment in National Highway 3 at Longtan Township, Taoyuan County, Taiwan (the VD is marked by $\times$ and the BS is marked by o)

two speeds is larger than a threshold $V_{s}$ ), then this technique should not be used. The details are described below.

Near-History Compensation Technique. The average speed of the target road segment is computed as

$$
v(\tau) \leftarrow \begin{cases}W v(\tau)+(1-W) v(\tau-\Delta t), & \text { for }|v(\tau)-v(\tau-\Delta t)|<V_{s} \\ v(\tau), & \text { for }|v(\tau)-v(\tau-\Delta t)| \geq V_{s}\end{cases}
$$

where $0 \leq W \leq 1$ is a weighting factor, $v(\tau-\Delta t)$ is the speed of the preceding timeslot of $\Delta \tau$, and $V_{s}$ is the threshold to detect the significant traffic change.

In (7), a larger $W$ value means that the past speed $v(\tau-\Delta t)$ has less effect on the current speed $v(\tau)$. 


\section{$6 \quad$ Numerical Examples}

This section compares the vehicle speeds derived from the LCH scheme with those measured by the VD scheme. We have obtained the vehicle speed data of National Highway 3 at Longtan Township, Taoyuan County, Taiwan (see Figure 4 (a)). The speed data were published by Ministry of Transportation and Communications, Taiwan, which were measured by the VD at the $66 \mathrm{~km}$ of the highway (see Figure 4 (b)). The Wideband Code Division Multiple Access (WCDMA) base stations are deployed along the highway. From a sector (cell) of a BS at Longtan Township (about $66.8 \mathrm{~km}$ of the highway, which is about 800 meters away from the VD; see Figure 4 (c)), we utilize the LCH scheme with filtering techniques 1 and 2, far-history and near-history compensation techniques to estimate the vehicle speeds from a cell of length $x=1 \mathrm{~km}$ and $\Delta t=1$ hour. The $x$ length is obtained through measurement $[16,17]$. We use the average of the numbers of handovers into the cell and out of the cell to estimate the speeds. Our experiments indicate that using the average of the numbers of handover-in and handover-out has better accuracy for the LCH scheme. To eliminate the effect of the ping-pong effect, we adopt the algorithm in [9] to filter out the inter-handover intervals which are less than 10 seconds. In this paper, we consider $K_{h}=10$, $V_{s}=40 \mathrm{~km} /$ hour, and $W=0.5$.

Let $v_{s}$ be the vehicle speeds measured by the $\mathrm{VD}(s=V D)$ and computed by the LCH scheme $(s=L C H)$, respectively. Figures 5 (a) and 5 (b) plot $v_{s}$ from 8:00 to 20:00 on September 16, 2011, in the northbound and southbound directions, respectively. In these figures, both the VD and the LCH approaches indicated that the traffic did not significantly vary during 8:00 to 20:00 (i.e., $v_{s}>75 \mathrm{~km} /$ hour). Figures 6 (a) and 6 (b) plot $v_{s}$ from 8:00 to 20:00 on September 25, 2011, in the northbound and southbound directions, respectively. In Figure 6 (a), both approaches captured a traffic jam occurred from 18:00 to 20:00 (i.e., $v_{s}<60 \mathrm{~km} /$ hour). In Figure 6 (b), both approaches indicated that the traffic did not significantly vary during 8:00 to 20:00 (i.e., $v_{s}>75 \mathrm{~km} /$ hour). Figures 5 and 6 show that 


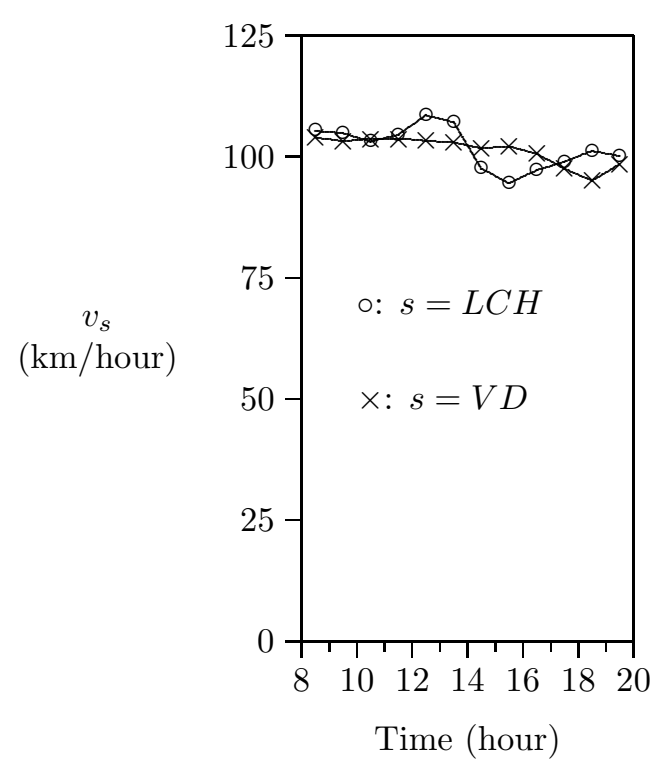

(a) Northbound

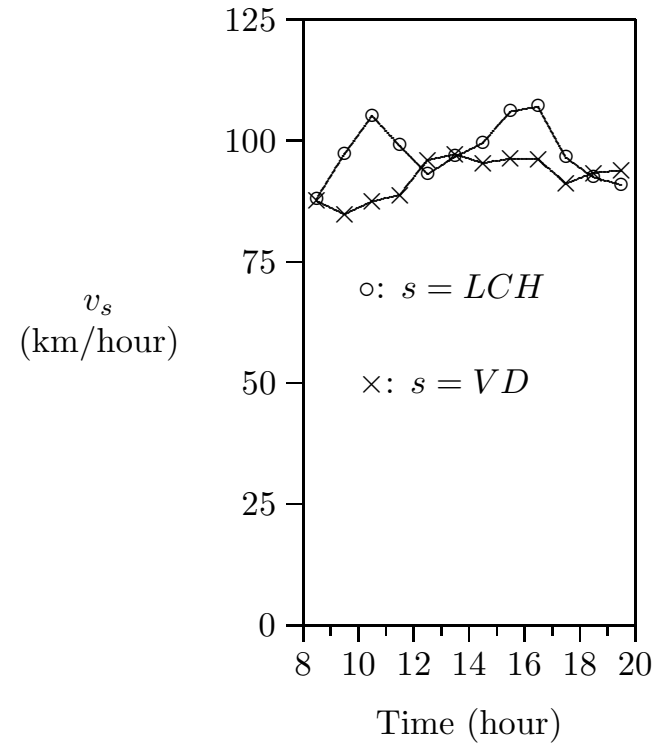

(b) Southbound

Figure 5: Vehicle Speeds of the VD and the LCH scheme on September 16, $2011(\Delta t=1$ hour and $x=1 \mathrm{~km}$ )

the trends of speeds of the LCH scheme are consistent with those of the VD scheme in both directions. We further define the discrepancy $\epsilon$ as

$$
\epsilon=\left|\frac{v_{L C H}-v_{V D}}{v_{V D}}\right|
$$

Based on Figures 5 and 6 , Figure 7 plots the $\epsilon$ curves in the observation time periods. The figure indicates that $\epsilon$ are reasonally small in most cases. When the traffic jam occurs (in Figure 7 (b), the o curve during 19:00 to 20:00), $\epsilon$ is slightly larger. The reason is that when the traffic jam occurs, the cell residence times of the vehicles are typically longer, and (5) already implies that the LCH scheme has lower accuracy for longer cell residence times.

We have also collected $\gamma(\tau)$ and $\rho(\tau)$ statistics during 8:00 to 20:00 over 49 days (September 13, 2011 to October 31, 2011) from the mobile telecommunications network. Based on the observed data, our study indicates that $E[\epsilon]$ is $14.46 \%$ if none of the techniques in Section 5 is applied, $E[\epsilon]$ is reduced to $12.7 \%$ if filtering techniques 1 and 2 are applied, and $E[\epsilon]$ is further reduced to $7.51 \%$ if both filtering and compensation techniques are all applied. 


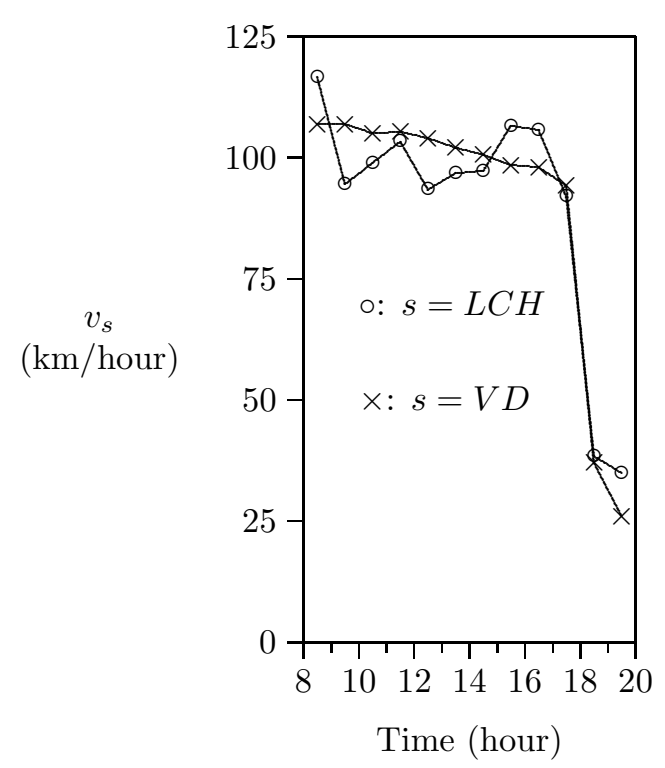

(a) Northbound

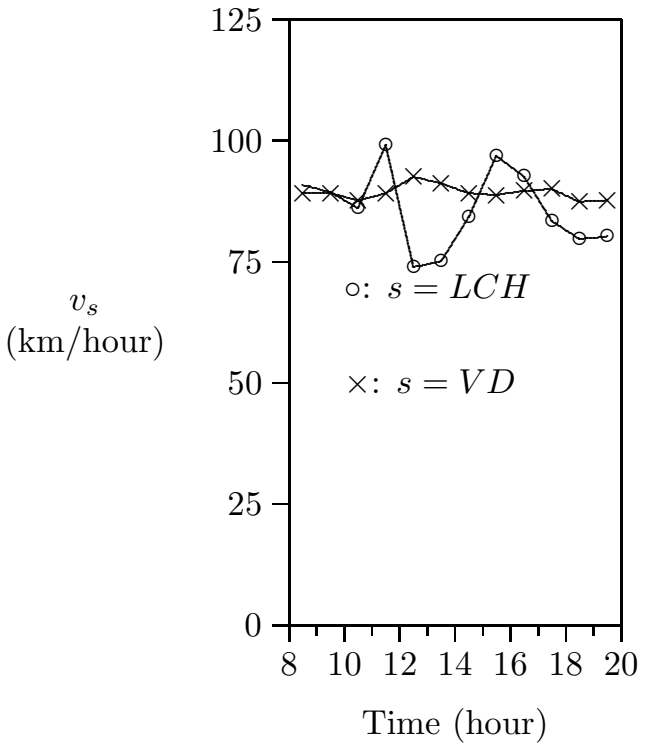

(b) Southbound

Figure 6: Vehicle Speeds of the VD and the LCH scheme on September 25, $2011(\Delta t=1$ hour and $x=1 \mathrm{~km}$ )

\section{Conclusions}

This paper extended the previously proposed Lin-Chang-Huangfu (LCH) scheme [1, 2] to derive the vehicle speeds. The bias analysis indicated that the LCH scheme is an appropriate approach for vehicle speed estimation (as compared with the pedestrian speed estimation). We further utilized several techniques to improve the accuracy of the LCH scheme, and then compared the vehicle speeds derived from the improved LCH scheme with those measured by a Vehicle Detector (VD) installed by the government. The comparison study showed that the trends of speeds derived from the LCH and the VD schemes are consistent, and the discrepancies between these two schemes are reasonably small in most cases, where the average discrepancy $E[\epsilon]$ is $7.51 \%$. Our study indicates that the LCH scheme can appropriately capture the vehicle speeds of the roads, and can avoid expensive deployment costs of existing approaches (e.g., sensor deployment of the VD scheme).

One potential issue of methods based on MSC statistical data is that it only applies to cell phones actually connected. This typically will be only a fraction of the cell phones within 

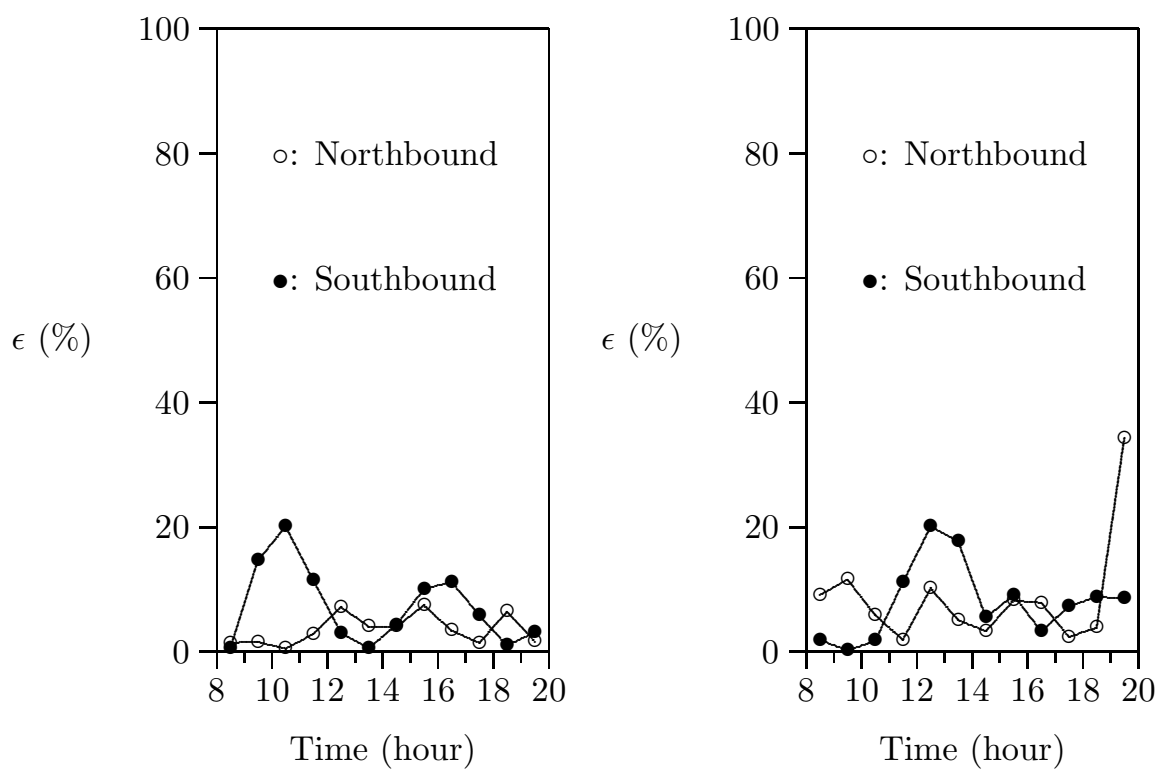

(a) The Experiment on September 16, (b) The Experiment on September 25, 2011 2011

Figure 7: Discrepancy between the LCH and the VD schemes $(\Delta t=1$ hour and $x=1 \mathrm{~km})$

the cell. Our study indicated that the number of the connected phones is reasonably large to produce data for our measurements and compensation techniques. Another potential issue is the selection of the time interval for statistical data collection. One may question that speed calculated in a period of 15 minutes or longer is not real-time. Our experience indicates that the 15-minute interval (or even longer) is appropriate. The traffic information broadcast from the government in Taiwan uses the 15-minute interval or longer. Many GVP approaches also use similar intervals to report the traffic information.

In the future, we will investigate the speed estimation in the Long Term Evolution (LTE) environment, and evaluate the performance of the LCH scheme based on packet-switched data connections. We will also develop more techniques to enhance the accuracy of the speed estimation for several scenarios (e.g., parallel road effect, few LA crossings, tourist effect, and so on). 


\section{A Detailed Proof of Theorem 1}

This appendix describes the proof for Theorem 1. We first prove the following facts.

Fact 1. Assume that $t_{c}$ is exponentially distributed and $t_{m}$ has an arbitrary density function $f_{m}\left(t_{m}\right)$ with the rate $\eta$. If the timeslot $\Delta \tau$ has an arbitrary distribution with the mean $\frac{1}{\delta}$, then $E[\beta(\tau)]=\frac{\lambda}{\mu}$ and $E[\gamma(\tau)]=\frac{\lambda \eta}{\delta \mu}$.

Proof: Consider the start time of timeslot $\Delta \tau$ (e.g., $t_{5}$ in Figure 2). This time point observes the call activities of cell $i$. The expected number $E[\beta(\tau)]$ of existing calls is proportional to $E[\alpha(\tau)]$ and the expected call holding time $\frac{1}{\mu}$, and is inversely proportional to the expected length $E[\Delta \tau]$ of timeslot. Therefore, we can express $E[\beta(\tau)]$ as

$$
E[\beta(\tau)]=\frac{E[\alpha(\tau)](1 / \mu)}{E[\Delta \tau]}=\frac{\lambda}{\mu}
$$

Intuitively, if $\frac{1}{\mu}<E[\Delta \tau]$, then $\frac{1}{\mu E[\Delta \tau]}$ is the probability that a call is an existing call in $\Delta \tau$. Then the total number of existing calls $E[\beta(\tau)]$ is $E[\alpha(\tau)]$ times $\frac{1}{\mu E[\Delta \tau]}$, which results in (9).

Obviously, $E[\gamma(\tau)]$ can be expressed as $E[\alpha(\tau)]$ times the average number of handovers of a call. Due to the memoryless property of the exponential distribution, the residual call holding time (e.g., $\tau_{c}=t_{15}-t_{13}$ in Figure 2 ) of a handover call is also exponential with the same mean $\frac{1}{\mu}$. Let the distribution function of $t_{m}$ be $F_{m}\left(t_{m}\right)$. Let $\tau_{m}$ (e.g., $\tau_{m}=t_{13}-t_{11}$ in Figure 2) be the residual cell residence time. According to the residual life theorem [13], the density function of $\tau_{m}$ is $\frac{1-F_{m}\left(t_{m}\right)}{(1 / \eta)}=\left[1-F_{m}\left(t_{m}\right)\right] \eta$. Note that the measured $t_{c}$ periods are carried call holding times. Therefore, if a handover occurs, a radio channel is available in the new cell, and the handover is always successful. Define $P_{i}$ as the probability that a new call completes after it has handed over $i$ times. Define $q_{1}=\operatorname{Pr}\left[t_{c}>\tau_{m}\right]$ as the probability that a new call hands over, and define 
$q_{2}=\operatorname{Pr}\left[\tau_{c}>t_{m}\right]$ as the probability that a call hands over under the condition that it has handed over before. Let $P_{i}=q_{1} q_{2}^{i-1}\left(1-q_{2}\right)$ where $i \geq 1$. Then the average number of handovers of a call can be expressed as

$$
\sum_{i=1}^{\infty} i P_{i}=\sum_{i=1}^{\infty} i q_{1} q_{2}^{i-1}\left(1-q_{2}\right)=\frac{q_{1}}{1-q_{2}}
$$

In (10), probability $q_{1}$ is derived as

$$
\begin{aligned}
q_{1}=\operatorname{Pr}\left[t_{c}>\tau_{m}\right] & =\int_{\tau_{m}=0}^{\infty} \int_{t_{c}=\tau_{m}}^{\infty} \mu e^{-\mu t_{c}}\left[1-F_{m}\left(\tau_{m}\right)\right] \eta d t_{c} d \tau_{m} \\
& =\int_{\tau_{m}=0}^{\infty} e^{-\mu \tau_{m}}\left[1-F_{m}\left(\tau_{m}\right)\right] \eta d \tau_{m}
\end{aligned}
$$

and $1-q_{2}$ can be expressed as

$$
\begin{aligned}
1-q_{2}=\operatorname{Pr}\left[\tau_{c}<t_{m}\right] & =\int_{\tau_{c}=0}^{\infty} \int_{t_{m}=\tau_{c}}^{\infty} \mu e^{-\mu \tau_{c}} f_{m}\left(t_{m}\right) d t_{m} d \tau_{c} \\
& =\int_{\tau_{c}=0}^{\infty} \mu e^{-\mu \tau_{c}}\left[1-F_{m}\left(\tau_{c}\right)\right] d \tau_{c} \\
& =\left(\frac{\mu}{\eta}\right) q_{1}
\end{aligned}
$$

From (11) and (12), the average number of handovers of a call is $\frac{q_{1}}{1-q_{2}}=\frac{\eta}{\mu}$. In a typical vehicle environment, the average number of handovers of a call is between 2 and 3 . The expected number $E[\gamma(\tau)]$ can be derived as

$$
E[\gamma(\tau)]=E[\alpha(\tau)]\left(\frac{\eta}{\mu}\right)=\frac{\lambda \eta}{\delta \mu}
$$

From the proof above, the derivation of $E[\alpha(\tau)], E[\beta(\tau)]$, and $E[\gamma(\tau)]$ are independent of the $\Delta \tau$ distribution.

Based on Fact 1, we have Facts 2 and 3. 
Fact 2. Assume that both $t_{c}$ and $\Delta \tau$ are exponentially distributed with the means $\frac{1}{\mu}$ and $\frac{1}{\delta}$, and $t_{m}$ has an arbitrary density function $f_{m}\left(t_{m}\right)$ with the rate $\eta$. Then $E[\rho(\tau)]=\frac{\lambda}{\delta \mu}$.

Proof: As defined in Fact 1, let the residual life of $t_{c}$ be $\tau_{c}$, and the residual life of $t_{m}$ be $\tau_{m}$. For timeslot $\Delta \tau$ of cell $i, \rho(\tau)$ is contributed by the conversation minutes of new, existing, and handover calls. Consider the new call for UE 3 during the timeslot $\Delta \tau$ in Figure 2. This call arrives at $t_{11}$ with $t_{c}=t_{15}-t_{11}$. At $t_{11}$, the residual life of UE 3's cell residence time is $\tau_{m}=t_{13}-t_{11}$. Since the call arrivals are a Poisson Process, $t_{11}$ is a random observer of UE 3's cell residence time $t_{m}$. The time point $t_{11}$ is also a random observer of $\Delta \tau$. From the residual life theorem and the memoryless property of the exponential distribution, the residual life $\Delta \tau_{n}$ of $\Delta \tau$ seen by $t_{11}$ is also exponentially distributed with the mean $\frac{1}{\delta}$. Therefore, the expected call minutes $E\left[\rho_{n}(\tau)\right]$ of this new call in $\Delta \tau$ is

$$
\begin{aligned}
E\left[\rho_{n}(\tau)\right] & =E\left[\min \left(t_{c}, \tau_{m}, \Delta \tau_{n}\right)\right] \\
& =E\left[\min \left(\min \left(t_{c}, \Delta \tau_{n}\right), \tau_{m}\right)\right]
\end{aligned}
$$

Clearly, random variable $x_{n}=\min \left(t_{c}, \Delta \tau_{n}\right)$ is exponentially distributed with the rate $\mu+\delta$. Let the Laplace transform of $f_{m}\left(t_{m}\right)$ be $f_{m}^{*}\left(t_{m}\right)$. From the derivation in [14], (14) is re-written as

$$
E\left[\rho_{n}(\tau)\right]=E\left[\min \left(x_{n}, \tau_{m}\right)\right]=\frac{1}{\mu+\delta}-\left[\frac{\eta}{(\mu+\delta)^{2}}\right]\left[1-f_{m}^{*}(\mu+\delta)\right]
$$

Now consider the existing call for UE 1 during $\Delta \tau$ in Figure 2. This call arrives at $t_{4}$. At $t_{5}$, the residual call holding time is $\tau_{c}=t_{16}-t_{5}$, and the residual life of UE 1 's cell residence time is $\tau_{m}=t_{18}-t_{5}$. Since $t_{5}$ can be considered as a random observer of UE 1's call holding time $t_{c}, \tau_{c}$ is exponentially distributed with the mean $\frac{1}{\mu}$. Then the 
expected call minutes $E\left[\rho_{e}(\tau)\right]$ of this existing call in $\Delta \tau$ is

$$
E\left[\rho_{e}(\tau)\right]=E\left[\min \left(\tau_{c}, \tau_{m}, \Delta \tau\right)\right]
$$

Since both $\tau_{c}$ and $\Delta \tau$ are exponentially distributed with the rates $\mu$ and $\delta, E\left[\rho_{e}(\tau)\right]$ can be expressed as (15); in other words, $E\left[\rho_{e}(\tau)\right]=E\left[\rho_{n}(\tau)\right]$.

Finally, consider the handover call for UE 4 during $\Delta \tau$ in Figure 2. This user moves to cell $i$ at $t_{9}$ with the cell residence time $t_{m}=t_{17}-t_{9}$. At $t_{9}$, the residual life of the call holding time is $\tau_{c}=t_{14}-t_{9}$. Since $t_{9}$ is a random observer of UE 4 's call holding time $t_{c}$, which is exponentially distributed with the mean $\frac{1}{\mu}, \tau_{c}$ has the same distribution as $t_{c}$. The time point $t_{9}$ is also a random observer of $\Delta \tau$, and the residual life $\Delta \tau_{h}$ of $\Delta \tau$ seen by $t_{9}$ is also exponentially distributed with the mean $\frac{1}{\delta}$. Therefore the expected call minutes $E\left[\rho_{h}(\tau)\right]$ of this handover call in $\Delta \tau$ is

$$
\begin{aligned}
E\left[\rho_{h}(\tau)\right] & =E\left[\min \left(\tau_{c}, t_{m}, \Delta \tau_{h}\right)\right] \\
& =E\left[\min \left(\min \left(\tau_{c}, \Delta \tau_{h}\right), t_{m}\right)\right]
\end{aligned}
$$

Let $x_{h}=\min \left(\tau_{c}, \Delta \tau_{h}\right)$, which is exponentially distributed with the rate $\mu+\delta$. Then similar to the derivation of (15), (16) is re-written as

$$
E\left[\rho_{h}(\tau)\right]=E\left[\min \left(x_{h}, t_{m}\right)\right]=\left(\frac{1}{\mu+\delta}\right)\left[1-f_{m}^{*}(\mu+\delta)\right]
$$


From (4), (15), (17), and Fact 1, $E[\rho(\tau)]$ is expressed as

$$
\begin{aligned}
E[\rho(\tau)]= & E[\alpha(\tau)] E\left[\rho_{n}(\tau)\right]+E[\beta(\tau)] E\left[\rho_{e}(\tau)\right]+E[\gamma(\tau)] E\left[\rho_{h}(\tau)\right] \\
= & (E[\alpha(\tau)]+E[\beta(\tau)])\left\{\frac{1}{\mu+\delta}-\left[\frac{\eta}{(\mu+\delta)^{2}}\right]\left[1-f_{m}^{*}(\mu+\delta)\right]\right\} \\
& +E[\gamma(\tau)]\left\{\left(\frac{1}{\mu+\delta}\right)\left[1-f_{m}^{*}(\mu+\delta)\right]\right\} \\
= & \frac{\lambda}{\delta \mu}
\end{aligned}
$$

Fact 3. Let $\Delta \tau$ be a fixed value $\frac{1}{\delta}$. If the call holding time $t_{c}$ and the cell residence time $t_{m}$ are exponentially distributed, then $E[\rho(\tau)]=\frac{\lambda}{\delta \mu}$.

Proof: Like the proof of Fact 2, $\rho(\tau)$ is contributed by the conversation minutes of new, existing, and handover calls. The expected call minutes $E\left[\rho_{n}(\tau)\right]$ of the new call in $\Delta \tau$ is

$$
E\left[\rho_{n}(\tau)\right]=E\left[\min \left(y_{n}, \Delta \tau_{n}\right)\right]=E\left[\min \left(\min \left(t_{c}, \tau_{m}\right), \Delta \tau_{n}\right)\right]
$$

which is the same as (14) except that $\Delta \tau_{n}$ is uniformly distributed over the interval $\left[0, \frac{1}{\delta}\right]$ with the density function $\delta$. Since both $t_{c}$ and $\tau_{m}$ are exponentially distributed with the rates $\mu$ and $\eta$, respectively, random variable $y_{n}=\min \left(t_{c}, \tau_{m}\right)$ is exponentially distributed with the rate $\mu+\eta$, and (18) is re-written as

$$
\begin{aligned}
E\left[\rho_{n}(\tau)\right]= & \int_{\Delta \tau_{n}=0}^{\frac{1}{\delta}} \int_{y_{n}=0}^{\Delta \tau_{n}} \delta y_{n}(\mu+\eta) e^{-(\mu+\eta) y_{n}} d y_{n} d \Delta \tau_{n} \\
& +\int_{\Delta \tau_{n}=0}^{\frac{1}{\delta}} \int_{y_{n}=\Delta \tau_{n}}^{\infty} \delta \Delta \tau_{n}(\mu+\eta) e^{-(\mu+\eta) y_{n}} d y_{n} d \Delta \tau_{n} \\
= & \left(\frac{1}{\mu+\eta}\right)\left[1-\frac{\delta}{\mu+\eta}+\frac{\delta e^{-\left(\frac{\mu+\eta}{\delta}\right)}}{\mu+\eta}\right]
\end{aligned}
$$


Now consider the expected call minutes $E\left[\rho_{h}(\tau)\right]$ of the handover call in $\Delta \tau$ is

$$
E\left[\rho_{h}(\tau)\right]=E\left[\min \left(\tau_{c}, t_{m}, \Delta \tau_{h}\right)\right]
$$

Both $\tau_{c}$ and $t_{m}$ are exponentially distributed with the rates $\mu$ and $\eta$, and $\Delta \tau_{h}$ is uniformly distributed over the interval $\left[0, \frac{1}{\delta}\right]$. Therefore, (20) can be expressed as (18); in other words, $E\left[\rho_{h}(\tau)\right]=E\left[\rho_{n}(\tau)\right]$. The expected call minutes $E\left[\rho_{e}(\tau)\right]$ of the existing call in $\Delta \tau$ is

$$
E\left[\rho_{e}(\tau)\right]=E\left[\min \left(y_{e}, \frac{1}{\delta}\right)\right]=E\left[\min \left(\min \left(\tau_{c}, \tau_{m}\right), \frac{1}{\delta}\right)\right]
$$

where $y_{e}=\min \left(\tau_{c}, \tau_{m}\right)$ is exponentially distributed with the rate $\mu+\eta$, and (21) is re-written as

$$
\begin{aligned}
E\left[\rho_{e}(\tau)\right] & =\int_{y_{e}=0}^{\frac{1}{\delta}} y_{e}(\mu+\eta) e^{-(\mu+\eta) y_{e}} d y_{e}+\int_{y_{e}=\frac{1}{\delta}}^{\infty}\left(\frac{1}{\delta}\right)(\mu+\eta) e^{-(\mu+\eta) y_{e}} d y_{e} \\
& =\left(\frac{1}{\mu+\eta}\right)\left[1-e^{-\left(\frac{\mu+\eta}{\delta}\right)}\right]
\end{aligned}
$$

From (4), (19), (22) and Fact 1, $E[\rho(\tau)]$ is expressed as

$$
\begin{aligned}
E[\rho(\tau)]= & E[\alpha(\tau)] E\left[\rho_{n}(\tau)\right]+E[\beta(\tau)] E\left[\rho_{e}(\tau)\right]+E[\gamma(\tau)] E\left[\rho_{h}(\tau)\right] \\
= & (E[\alpha(\tau)]+E[\gamma(\tau)])\left(\frac{1}{\mu+\eta}\right)\left[1-\frac{\delta}{\mu+\eta}+\frac{\delta e^{-\left(\frac{\mu+\eta}{\delta}\right)}}{\mu+\eta}\right] \\
& +E[\beta(\tau)]\left(\frac{1}{\mu+\eta}\right)\left[1-e^{-\left(\frac{\mu+\eta}{\delta}\right)}\right] \\
= & \frac{\lambda}{\delta \mu}
\end{aligned}
$$

Fact 4. Assume that $\Delta \tau, t_{c}$ and $t_{m}$ have arbitrary distributions with the means $\frac{1}{\delta}, \frac{1}{\mu}$ and $\frac{1}{\eta}$, respectively. Then $E[\gamma(\tau)] \approx \frac{\lambda \eta}{\delta \mu}$. 
Proof: Consider a long observation time period $t \gg \frac{1}{\delta}, \frac{1}{\mu}$. Let $t^{*}$ be the time which is occupied by the calls to a user during period $t$. Then there are $\left\lceil\frac{t^{*}}{(1 / \mu)}\right\rceil=\left\lceil\mu t^{*}\right\rceil$ call arrivals within $t$. Note that the user is expected to move across cells $\left\lfloor\frac{t}{(1 / \eta)}\right\rfloor$ times in period $t$, and among these crossings, $\left\lfloor\frac{t^{*}}{(1 / \eta)}\right\rfloor$ of them occur during conversations. Therefore the number of handovers is $\left\lfloor\frac{t^{*}}{(1 / \eta)}\right\rfloor=\left\lfloor\eta t^{*}\right\rfloor$, and the average number of handovers of a call is $E\left[n_{1}\right]=\frac{\left\lfloor\eta t^{*}\right\rfloor}{\left[\mu t^{*}\right\rceil}$. Clearly, $\lim _{t^{*} \rightarrow \infty} E\left[n_{1}\right]=\frac{\eta}{\mu}$, and therefore if $t$ is long enough, we have

$$
E[\gamma(\tau)]=E[\alpha(\tau)] E\left[n_{1}\right] \approx \frac{\lambda \eta}{\delta \mu}
$$

The result of Fact 4 is consistent with that of Fact 1 .

Fact 5. Assume that $\Delta \tau=\frac{1}{\delta}$ is fixed, and $t_{c}$ and $t_{m}$ have arbitrary distributions with the means $\frac{1}{\mu}$ and $\frac{1}{\eta}$, respectively. Let $t$ be the observation period. Then $\lim _{t \rightarrow \infty} E[\rho(\tau)]=$ $\frac{\lambda}{\delta \mu}$.

Proof: We assume that the UEs are moving around $n_{2}$ cells, and their behaviors are observed in a long time period $t \gg \frac{n_{2}}{\delta}, \frac{n_{2}}{\mu}$. Since the UEs will not leave $n_{2}$ cells, handovers of these calls (and therefore the $t_{m}$ distribution) will not affect the statistics of call minutes. There are $\left\lceil\lambda t n_{2}\right\rceil$ calls arrive in $n_{2}$ cells during the period $t$. Some of the calls are initiated before the beginning of $t$, which are not counted (we call it the initial effect). Some of the counted calls do not complete at the end of $t$ (we call it the end effect). Let $t_{c, i}$ be the call holding time of the $i$-th call, and $f_{c}\left(t_{c, i}\right)$ be the density function of the call holding time of the $i$-th call with the rate $\mu$. Then the measured call minutes $\theta$ of these $n_{2}$ cells during $t$ can be approximated as

$$
\theta=\sum_{i=1}^{\left\lceil\lambda t n_{2}\right\rceil} \int_{t_{c, i=0}}^{\infty} t_{c, i} f_{c}\left(t_{c, i}\right) d t_{c, i}=\frac{\left\lceil\lambda t n_{2}\right\rceil}{\mu}
$$


There are total $n_{2}\left\lceil\frac{t}{(1 / \delta)}\right\rceil=\left\lceil n_{2} t \delta\right\rceil$ timeslots. Since $E[\rho(\tau)]$ is the expected call minutes of traffic in $\Delta \tau$ of cell $i$, it can be approximated as $E[\rho(\tau)] \approx \frac{\theta}{\left\lceil n_{2} t \delta\right\rceil}$. If $t$ becomes large, the initial and the end effects become insignificant, and

$$
\lim _{t \rightarrow \infty} E[\rho(\tau)]=\frac{\lambda}{\delta \mu}
$$

Note that the result in Fact 5 is the same as those in Facts 2 and 3. These results are also consistent with the Erlang equation, which defines the traffic per minute as $\frac{\lambda}{\mu}$. Therefore $\frac{\lambda}{\delta \mu}$ is the traffic in timeslot $\Delta \tau$.

Theorem 1. Assume that $t_{c}$ and $t_{m}$ have arbitrary distributions with the means $\frac{1}{\mu}$ and $\frac{1}{\eta}$, respectively. If timeslot $\Delta \tau=\frac{1}{\delta}$ is fixed, and we observe the call activities for a long period $t$, then $b_{t_{m}}\left(t_{m}^{*}(\tau)\right)=\frac{\mu \delta}{\lambda \eta^{2}}$.

Proof: Suppose that we observe the call activities for a long period $t \rightarrow \infty$. Since the measurements of the LCH scheme are conducted in $\Delta \tau$, from (23) and (24), both $E[\gamma(\tau)]$ and $E[\rho(\tau)]$ go to infinity if $\frac{1}{\delta}$ becomes infinity, and (1) cannot be used to compute $t_{m}^{*}(\tau)$. To fix this problem, let $c_{1}=\frac{E[\rho(\tau)]}{(1 / \delta)}=\frac{\lambda}{\mu}$ and $c_{2}=\frac{E[\gamma(\tau)]}{(1 / \delta)}=\frac{\lambda \eta}{\mu}$. Then we can re-write $(1)$ as a function $f(r, s)$ such that

$$
t_{m}^{*}(\tau)=\left.f(r, s)\right|_{r=c_{1}, s=c_{2}}=\left.\left(\frac{r}{s}\right)\right|_{r=c_{1}, s=c_{2}}
$$


To compute (25), we use the Taylor series for $f(r, s)$ around the point $\left(c_{1}, c_{2}\right)$, which is

$$
\begin{aligned}
f(r, s)= & f\left(c_{1}, c_{2}\right)+\left[\left.\frac{\partial f(r, s)}{\partial r}\right|_{r=c_{1}, s=c_{2}}\right]\left(r-c_{1}\right) \\
& +\left[\left.\frac{\partial f(r, s)}{\partial s}\right|_{r=c_{1}, s=c_{2}}\right]\left(s-c_{2}\right)+\left(\frac{1}{2}\right)\left[\left.\frac{\partial^{2} f(r, s)}{\partial r^{2}}\right|_{r=c_{1}, s=c_{2}}\right]\left(r-c_{1}\right)^{2} \\
& +\left[\left.\frac{\partial}{\partial s}\left(\frac{\partial f(r, s)}{\partial r}\right)\right|_{r=c_{1}, s=c_{2}}\right]\left(r-c_{1}\right)\left(s-c_{2}\right) \\
& +\left(\frac{1}{2}\right)\left[\left.\frac{\partial^{2} f(r, s)}{\partial s^{2}}\right|_{r=c_{1}, s=c_{2}}\right]\left(s-c_{2}\right)^{2}+\cdots
\end{aligned}
$$

Since we consider the $f(r, s)$ function around the point $\left(c_{1}, c_{2}\right)$ and the expected value $E[r]=c_{1}$ and $E[s]=c_{2}$, we have

$$
E\left[r-c_{1}\right]=0, E\left[s-c_{2}\right]=0 \text { and } E\left[\left(r-c_{1}\right)\left(s-c_{2}\right)\right]=0
$$

Also, from (25)

$$
\left.\frac{\partial^{2} f(r, s)}{\partial r^{2}}\right|_{r=c_{1}, s=c_{2}}=0 \text { and }\left.\frac{\partial^{2} f(r, s)}{\partial s^{2}}\right|_{r=c_{1}, s=c_{2}}=\frac{2 c_{1}}{c_{2}^{3}}
$$

From (27) and (28), the expectation of (26) can be written as

$$
\begin{aligned}
E[f(r, s)] & =f\left(c_{1}, c_{2}\right)+\left(\frac{1}{2}\right)\left[\left.\frac{\partial^{2} f(r, s)}{\partial s^{2}}\right|_{r=c_{1}, s=c_{2}}\right] E\left[\left(s-c_{2}\right)^{2}\right] \\
& =\frac{c_{1}}{c_{2}}+\left(\frac{c_{1}}{c_{2}^{3}}\right) E\left[\left(s-c_{2}\right)^{2}\right] \\
& =\frac{1}{\eta}+\left(\frac{\mu^{2}}{\lambda^{2} \eta^{3}}\right) E\left[\left(s-c_{2}\right)^{2}\right]
\end{aligned}
$$

In $(29), E\left[\left(s-c_{2}\right)^{2}\right]=\operatorname{Var}\left[\frac{\gamma(\tau)}{(1 / \delta)}\right]=\delta^{2} \operatorname{Var}[\gamma(\tau)]$. From $(13), \gamma(\tau)$ is a Poisson random variable with the mean $E[\gamma(\tau)]=\frac{\lambda \eta}{\delta \mu}$ and the variance $\operatorname{Var}[\gamma(\tau)]=\frac{\lambda \eta}{\delta \mu}$. Therefore, $(29)$ 
is re-written as

$$
\begin{aligned}
E[f(r, s)] & =\frac{1}{\eta}+\left(\frac{\mu^{2}}{\lambda^{2} \eta^{3}}\right) \operatorname{Var}\left[\frac{\gamma(\tau)}{\left(\frac{1}{\delta}\right)}\right] \\
& =\frac{1}{\eta}+\frac{\mu \delta}{\lambda \eta^{2}}
\end{aligned}
$$

Since the mean of $t_{m}$ is $\frac{1}{\eta}$, from $(3)$ and $(30), b_{t_{m}}\left(t_{m}^{*}(\tau)\right)$ is

$$
b_{t_{m}}\left(t_{m}^{*}(\tau)\right)=E\left[t_{m}^{*}(\tau)\right]-E\left[t_{m}\right]=\left[\frac{1}{\eta}+\frac{\mu \delta}{\lambda \eta^{2}}\right]-\frac{1}{\eta}=\frac{\mu \delta}{\lambda \eta^{2}}
$$

\section{Acknowledgement}

The authors would like to thank the four anonymous reviewers. Their comments have significantly improved the quality of this paper.

\section{References}

[1] Yi-Bing Lin, Ming-Feng Chang, and Chien-Chun Huang-Fu , "Derivation of cell residence times from the counters of mobile telecommunications switches," IEEE Transactions on Wireless Communications, 2011, vol. 10, no. 12, pp. 4048-4051.

[2] Yi-Bing Lin, Chien-Chun Huang-Fu, and Nabil Alrajeh, "Predicting human movement based on telecom's handoff in mobile networks," IEEE Transactions on Mobile Computing, in press.

[3] Chien-Chun Huang-Fu and Yi-Bing Lin, "Deriving Vehicle Speeds From Standard Statistics of Mobile Telecom Switches," IEEE Transactions on Vehicular Technology, 2012, vol. 61, no. 7, pp. 3337-3341. 
[4] Ren-Huang Liou, Yi-Bing Lin, Yu-Long Chang, and Ming-Feng Chang, "Deriving the vehicle speeds from mobile telecommunications network," the 12th International Conference on ITS Telecommunications (ITST), November 2012.

[5] Sang Jin Park, Tae Yong Kim, Sung Min Kang, and Kyung Heon Koo, "A novel signal processing technique for vehicle detection radar," in 2003 IEEE MTT-S International Microwave Symposium Digest, 2003, pp. 8-13.

[6] Anurak Poolsawat, Wasan Pattara-Atikom, and Boonchai Ngamwongwattana, "Acquiring road traffic information through mobile phones," in 8th International Conference on ITS Telecommunications (ITST), 2008, pp. 170-174.

[7] Ruey Long Cheu, Chi Xie, and Der-Horng Lee, "Probe vehicle population and sample size for arterial speed estimation," Computer-Aided Civil and Infrastructure Engineering, 2002, vol. 17, no. 1, pp. 5360.

[8] Chih-Yi Chiang, Ju-Yin Chuang, Jian-Kai Chen, Chia-Chen Hung, Wei-Hui Chen, and Kuen-Rong Lo, "Estimating instant traffic information by identifying handover patterns of UMTS signals," in 2011 14th International IEEE Conference on Intelligent Transportation Systems (ITSC), 2011, pp. 390-395.

[9] Kuen-Rong Lo, Chih-Yi Chiang, Ju-Yin Chuang, Jian-Kai Chen, Chia-Chen Hung, and Wei-Hui Chen, "Feasibility analysis of UMTS handover logs for traffic state estimation," in 2011 11th International Conference on ITS Telecommunications (ITST), 2011, pp. 684-690.

[10] Bon-Yeh Lin, Chi-Hua Chen, and Chi-Chun Lo, "A traffic information estimation model using periodic location update events from cellular network", Communications in Computer and Information Science, 2011, vol. 135, pp. 72-77.

[11] Yi-Bing Lin and Ai-Chun Pang, Wireless and Mobile All-IP Networks. John Wiley \& Sons, Inc., 2005. 
[12] Jay L. Devore, Probability and Statistics for Engineering and the Sciences. Duxbury Press, 2011.

[13] Leonard Kleinrock, Queueing Systems, Vol. I, Theory, Wiley, New York, 1976.

[14] Yi-Bing Lin, "Performance modeling for mobile telephone networks", IEEE Network, 1997, vol. 11, pp. 6368.

[15] Yi-Bing Lin and Imrich Chlamtac, Wireless and Mobile Network Architectures. John Wiley \& Sons, Inc., 2000.

[16] D. Gundlegard and J.-M. Karlsson, "Handover Location Accuracy for Travel Time Estimation in GSM and UMTS," IET Intelligent Transport Systems, 2009, vol. 3, no. 1, pp. 87-94.

[17] Hillel Bar-Gera, "Evaluation of a Cellular Phone-Based System for Measurements of Traffic Speeds and Travel Times: A Case Study from Israel," Transpiration. Research Part C: Emerging Technologies, 2007, vol. 15, no. 6, pp. 380-391.

[18] Danilo Valerio, Tobias Witek, Fabio Ricciato, Rene Pilz, and Werner Wiedermann, "Road traffic estimation from cellular network monitoring: a hands-on investigation," 2009 IEEE 20th International Symposium on Personal, Indoor and Mobile Radio Communications, 2009, pp. 3035-3039. 